\title{
Characterization of a synthetic single crystal diamond Schottky diode for radiotherapy electron beam dosimetry
}

\author{
C. Di Venanzio, Marco Marinelli, E. Milani, G. Prestopino, C. Verona, and G. Verona-Rinatia) \\ INFN-Dipartimento di Ingegneria Industriale, Università di Roma 'Tor Vergata', Via del Politecnico 1, \\ 00133 Roma, Italy \\ M. D. Falco, P. Bagalà, and R. Santoni \\ Department of Diagnostic Imaging, Molecular Imaging, Interventional Radiology and Radiotherapy, \\ Tor Vergata University General Hospital, Viale Oxford 81, 00133 Rome, Italy \\ M. Pimpinella \\ Istituto Nazionale di Metrologia delle Radiazioni Ionizzanti, ENEA-INMRI C R Casaccia, Via Anguillarese \\ 301, 00123 Roma, Italy
}

(Received 11 September 2012; revised 15 November 2012; accepted for publication 20 December 2012; published 24 January 2013)

Purpose: To investigate the dosimetric properties of synthetic single crystal diamond based Schottky diodes under irradiation with therapeutic electron beams from linear accelerators.

Methods: A single crystal diamond detector was fabricated and tested under 6, 8, 10, 12, and $15 \mathrm{MeV}$ electron beams. The detector performances were evaluated using three types of commercial detectors as reference dosimeters: an Advanced Markus plane parallel ionization chamber, a Semiflex cylindrical ionization chamber, and a $p$-type silicon detector. Preirradiation, linearity with dose, dose rate dependence, output factors, lateral field profiles, and percentage depth dose profiles were investigated and discussed.

Results: During preirradiation the diamond detector signal shows a weak decrease within $0.7 \%$ with respect to the plateau value and a final signal stability of $0.1 \%(1 \sigma)$ is observed after about $5 \mathrm{~Gy}$. A good linear behavior of the detector response as a function of the delivered dose is observed with deviations below $\pm 0.3 \%$ in the dose range from 0.02 to $10 \mathrm{~Gy}$. In addition, the detector response is dose rate independent, with deviations below $0.3 \%$ in the investigated dose rate range from 0.17 to $5.45 \mathrm{~Gy} / \mathrm{min}$. Percentage depth dose curves obtained from the diamond detector are in good agreement with the ones from the reference dosimeters. Lateral beam profile measurements show an overall good agreement among detectors, taking into account their respective geometrical features. The spatial resolution of solid state detectors is confirmed to be better than that of ionization chambers, being the one from the diamond detector comparable to that of the silicon diode. A good agreement within experimental uncertainties was also found in terms of output factor measurements between the diamond detector and reference dosimeters.

Conclusions: The observed dosimetric properties indicate that the tested diamond detector is a suitable candidate for clinical electron beam dosimetry. () 2013 American Association of Physicists in Medicine. [http://dx.doi.org/10.1118/1.4774360]

Key words: electron beam dosimetry, synthetic diamond detector

\section{INTRODUCTION}

Dosimetry in high energy electron beam radiotherapy treatments requires small volume, radiation hard, energy independent detectors. Conventional detectors recommended by international electron dosimetry protocols are air filled ionization chambers and silicon diodes. ${ }^{1-4}$ Ionization chambers provide accurate and highly reproducible dose measurements. However, beam quality and depth dependent ionization-to-dose conversion factors, such as stopping-power ratios and perturbation factors, as well as polarity effects and ion recombination corrections have to be accounted for when converting the ionization signal to dose measurement. ${ }^{2-4}$ In addition, spatial averaging effects due to the finite size of ionization chambers make them not suitable when high spatial resolution is required. ${ }^{5}$ Although being not tissue-equivalent, sil- icon diodes ( $p$-type) have been well established in clinical electron dosimetry due to their small sensitive volume, high sensitivity, and high spatial resolution. ${ }^{6-8}$ The silicon to water stopping power ratio is fairly constant for electron energies between 5 and $25 \mathrm{MeV}$ and, once calibrated against an ionization chamber, the diode response is assumed to represent the dose with no need of depth-dependent correction factors. ${ }^{2-4}$ However, the silicon to water stopping power ratio decreases with decreasing electron energy for energies lower than $5 \mathrm{MeV}$ with a variation of about $6 \%$ from 5 to $0.5 \mathrm{MeV}$. This may lead to significant dose overestimation in depthdose scans and lateral beam profiles, due to the abundance of low energy scattered electrons. In addition, dose rate dependence, angular dependence, and radiation damage may constitute a limit to the use of silicon diodes for clinical electron dosimetry. ${ }^{10}$ 
Diamond has long been considered the material of choice for dosimetric applications allowing fabrication of detectors with high sensitivity per unit volume, spatial resolution comparable to that of silicon diode detectors, low leakage current, and high radiation hardness. ${ }^{11-17}$ Moreover, the carbon to water stopping power ratio stays nearly constant over an electron energy range 1-20 MeV; thus, allowing direct measurement of dose distributions in tissue equivalent medium. ${ }^{1,5}$ Furthermore, unlike silicon diodes, the same diamond detector is expected to be suitable for both electron and photon dosimetry. ${ }^{5}$

Most studies on diamond application for megavoltage electron and photon radiotherapy beam dosimetry have been performed using PTW natural diamond detectors and are reported as comparative investigations with respect to reference detectors, i.e., ionization chambers and silicon diodes. ${ }^{5,16-21}$

Although natural diamonds are well assessed in the market, their use is limited by a number of drawbacks, such as high costs, long delivery time due to suitable stones selection, lack of response reproducibility among detectors, and high dose rate dependence, which implies the use of a sampledependent correction factor in dosimetric measurements. On the other hand, spotty results have been reported on synthetic high-pressure high-temperature (HPHT) (Refs. 15 and 22) and polycrystalline chemical vapor deposition (CVD) diamonds. ${ }^{23}$ Difficulties in controlling both type and amount of incorporated impurities in HPHT diamonds and the presence of structural defects in polycrystalline CVD diamonds seriously limit the production of detector-grade crystals and strongly affect the response stability and the overall dosimetric properties of detectors. Recently, the feasibility of high performance radiotherapy detectors based on synthetic single crystal diamond grown by means of CVD technique at the laboratories of the University of Rome "Tor Vergata" has been studied. ${ }^{24-26}$ In particular, the suitability of such detectors for the dosimetry of radiation therapy small photon beams has been investigated and very promising results have been pointed out. ${ }^{26,27}$

In this work the dosimetric performances of such detectors are evaluated in megavoltage clinical electron beams with energy in the range $6-15 \mathrm{MeV}$ and field size from $6 \times 6$ to $20 \times 20 \mathrm{~cm}^{2}$.

\section{MATERIALS AND METHOD}

\section{II.A. Linear accelerator}

Dosimetric measurements were carried out with $6,8,10$, 12 , and $15 \mathrm{MeV}$ electron beams generated by an Elekta Precise linear accelerator (Elekta Crawley, UK). Conventional beams, $6 \times 6,10 \times 10,14 \times 14$, and $20 \times 20 \mathrm{~cm}^{2}$ in size, were obtained by means of standard applicators attached to the head of the accelerator. The electron beam calibration, for each energy and applicator, was performed following the IAEA TRS-398 dosimetry protocol ${ }^{4}$ and using a PTW 34045 plane parallel ionization chamber (Advanced Markus, PTW Freiburg). The chamber was cross-calibrated in terms of absorbed dose to water against a reference Farmer chamber in the electron beam with the highest available energy
(15 MeV). Beam quality correction factors for reference dosimetry at lower beam energies were calculated according to the IAEA protocol. ${ }^{4}$ In particular, the Advanced Markus chamber can be considered a "well-guarded" chamber, since the electrode guard width is 2 times larger than the cavity height. A unity overall electron perturbation factor was, therefore, adopted. ${ }^{4,28}$

Depth ionization curves were converted to depth dose curves using the formula for the water-air stopping power ratios reported in Appendix II of the IAEA TRS-398 protocol. ${ }^{4}$ Reference absorbed dose to water measurements were performed, for each energy and applicator, at the reference depth as indicated by the IAEA protocol, and scaled to the depth of the dose maximum, $\mathrm{d}_{\max }$, according to the measured depth dose distributions. For each energy, the accelerator output with the $10 \times 10 \mathrm{~cm}^{2}$ reference applicator was of about $1 \mathrm{cGy} /$ monitor unit (MU) at $\mathrm{d}_{\max }$ for a source to surface distance, SSD, of $100 \mathrm{~cm}$.

During measurements relevant to the diamond detector characterization a cylindrical ionization chamber (Semiflex type 31010, PTW Freiburg) was placed in the path of the electron beam, in the periphery of the radiation field, and used as an external beam monitor. In each measurement session, the accelerator output stability was found to be within $\pm 0.5 \%$.

\section{II.B. Diamond detector}

A synthetic single crystal diamond detector (SCDD) was fabricated at Rome "Tor Vergata" University laboratories. The diamond plate consisted of a multilayered highly conductive $p$-type diamond/intrinsic diamond structure fabricated by a two-step microwave plasma enhanced CVD process on a commercial low cost $3.0 \times 3.0 \times 0.3 \mathrm{~mm}^{3}$ HPHT Ib single crystal diamond substrate. A thin rectifying circular aluminum contact, $2.2 \mathrm{~mm}$ in diameter, was thermally evaporated onto the intrinsic diamond surface, while annealed ohmic silver contacts were formed on the $p$-type diamond layer, which was used as a backing contact. Due to the formation of a builtin potential at the metal/intrinsic diamond interface, the device works as a Schottky-barrier photodiode. In all of the tests reported in the present paper it was operated in photovoltaicmode, i.e., with no external bias voltage applied. The detector sensitive volume, of about $3.8 \times 10^{-3} \mathrm{~mm}^{3}$, is thus defined by the depletion region extending through the whole thickness of the thin intrinsic diamond layer (1.0 $\pm 0.1 \mu \mathrm{m}$ thick), below the circular Al contact. A comprehensive description of the device fabrication process and of its physical properties and detection mechanism was reported in Ref. 29. The diamond detector was finally embedded at "Tor Vergata" laboratories, under PTW-Freiburg permission, in the very same waterproof PTW proprietary encapsulation kit used for the fabrication of type 60017 unshielded silicon diode detector. ${ }^{30}$ Figure 1 shows a picture of the SCDD and a schematic of the type 60017 silicon diode encapsulation design. The SCDD measurement point was assumed to be at the centre of the circular $\mathrm{Al}$ contact on the diamond surface, $0.8 \mathrm{~mm}$ from the detector tip. The diamond detector was positioned with its main axis parallel to the central beam axis (vertical orientation) to 
(a)

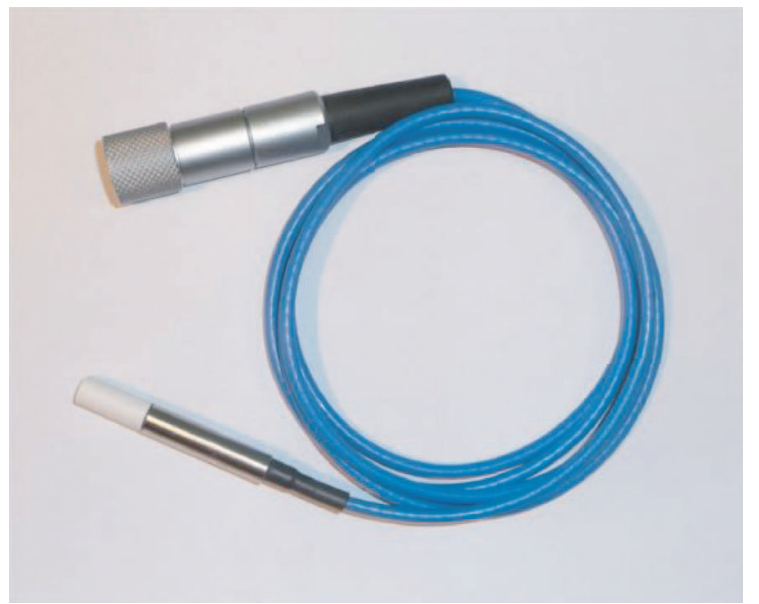

(b)

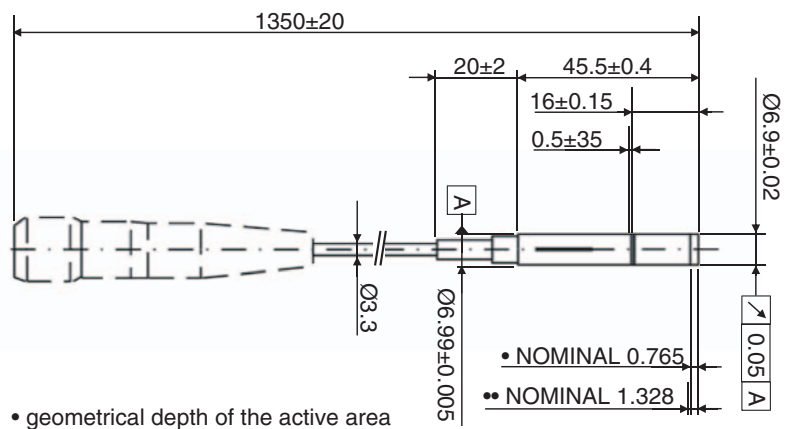

- water equivalent depth of the active area

FIG. 1. (a) Photo of the SCDD as embedded in the PTW-Freiburg type 60017 unshielded diode encapsulation casing and connected to the triaxial cable and connector. (b) Drawing of the dosimetry Diode E (type 60017) silicon detector from PTW-Freiburg radiation therapy detector catalogue.

minimize possible stem effects. During lateral beam profile measurements, the device was also positioned with its main axis perpendicular to the electron beam direction (horizontal orientation), in order to investigate the spatial resolution as a function of the device orientation. A sketch of all the SCDD configurations adopted in beam profile measurements, i.e., horizontal and vertical detector orientation for in-plane and cross-plane scans, is illustrated in Fig. 2. The scanning plane and the central beam axis (CAX), i.e., the electron beam direction, are also shown in the figure.

\section{II.C. Reference detectors and electrometers}

Three types of commercial detectors were used as reference dosimeters for the SCDD characterization: a plane parallel ionization chamber (Advanced Markus type 34045, PTW Freiburg), a cylindrical ionization chamber (Semiflex type 31010, PTW Freiburg), and a $p$-type silicon detector (Dosimetry Diode E type 60017, PTW Freiburg).

The Advanced Markus ionization chamber (AM-IC) is a plane-parallel chamber designed by PTW-Freiburg for relative and absolute high energy electron dosimetry. The chamber has a nominal sensitive volume of $20 \mathrm{~mm}^{3}$ with an air cavity height of $1 \mathrm{~mm}$ and a collecting electrode radius of $2.5 \mathrm{~mm}$. The chamber is equipped with a wide guard electrode ( $2 \mathrm{~mm}$ width), fulfilling the requirements for negligible scat-
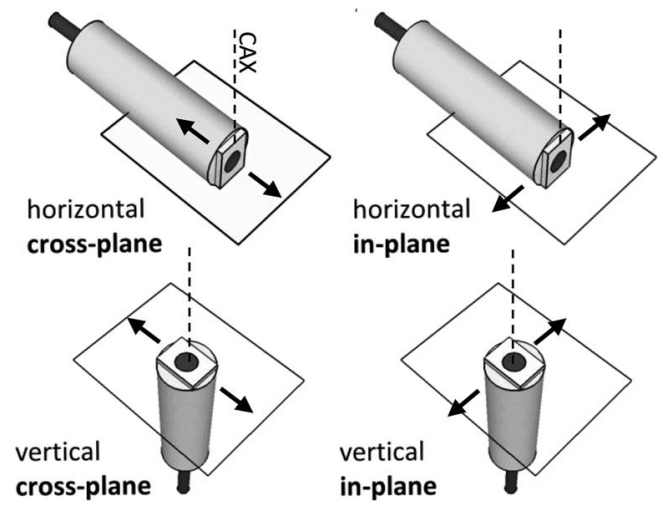

FIG. 2. Schematic diagram of the scanning procedures for lateral beam profile measurements by the SCDD in horizontal and vertical orientations with respect to the CAX.

tering perturbation effects in electron beams. ${ }^{4}$ According to dosimetry protocols ${ }^{2-4}$ the reference point for plane-parallel chambers is at the centre of the inside surface of the chamber entrance window. The water-equivalent thickness of the chamber wall including the waterproof cap was taken into account in positioning the chamber in water phantom. Therefore, the effective point of measurement in water was taken $1.06 \mathrm{~mm}$ below the top surface of the chamber protective cap. The chamber plane was oriented perpendicularly to the electron beam direction and a polarizing voltage of $+300 \mathrm{~V}$ was applied, according to user manual instructions. In this work the Advanced Markus chamber was used as reference dosimeter for the beam calibration, output factor, and central-axis depth dose measurements. It should be considered that literature data ${ }^{31}$ show that for some plane-parallel chambers ion recombination corrections can be overestimated using polarizing voltages exceeding $100 \mathrm{~V}$. For the chamber used in this work an ion recombination correction of $1.003 \pm 0.002$ was found for all the energies and depths considered. Therefore, the adopted polarizing voltage does not significantly affect the obtained results.

The Semiflex ionization chamber (SF-IC) is a cylindrical ion chamber with an internal radius of $2.75 \mathrm{~mm}$, a length of $6.5 \mathrm{~mm}$, and a $0.125 \mathrm{~cm}^{3}$ nominal sensitive volume. The chamber was oriented with its long axis perpendicular to the electron beam direction and a voltage bias of $+400 \mathrm{~V}$ was applied, according to user manual instructions. The effective point of measurement is placed $1.4 \mathrm{~mm}$ above the chamber axis and $4.5 \mathrm{~mm}$ from the chamber tip. Measurements of lateral beam profiles were carried out with the Semiflex chamber to be compared to those obtained by the SCDD.

The silicon diode ( $\mathrm{Si}-\mathrm{D}$ ) has a $1 \mathrm{~mm}^{2}$ circular, $30 \mu \mathrm{m}$ thick sensitive volume located on the detector axis, $0.8 \mathrm{~mm}$ below the detector front surface. The water-equivalent window thickness is $1.33 \mathrm{~mm}$. Following manufacturer's recommendations, the silicon detector was used in vertical orientation and no bias voltage was applied. The Si-D was used as a commercial solid state detector for comparative purposes in lateral beam profiles, depth dose, and output factor measurements.

A PTW Unidos E Universal Dosimeter was used for measurements of the SCDD signal stability, dose and dose rate 
dependence, and output factors. A PTW Tandem Dual Channel Electrometer was used for scanning measurements, i.e., lateral beam profiles and depth dose distributions.

\section{II.D. Dosimetric measurements}

The SCDD characterization included investigation of preirradiation dose, dose and dose rate dependence, capability of measuring depth dose distributions, transverse profiles, and output factors.

All measurements were performed in an MP3 PTW motorized water phantom at a SSD of $100 \mathrm{~cm}$. The zero depth of each detector point of measurement was set before data collection, as described in the following. At first the top surface of the detector was set precisely at the water surface and centered in the beam. Then a proper shift to the effective point of measurement was applied. Finally, the zero depth value was assigned to the resulting detector position in the computerized automatic scanning system. ${ }^{32}$

Measurements of preirradiation dose, dose, and dose rate dependence were performed using a $10 \mathrm{MeV}$ electron beam and a $10 \times 10 \mathrm{~cm}^{2}$ field size. In the preirradiation investigation, an overall dose of 20 Gy was delivered in 40 steps, $0.5 \mathrm{~Gy}$ each at a nominal dose rate of $3 \mathrm{~Gy} / \mathrm{min}$. Detector response linearity with dose was investigated in the dose range from 0.02 to $10 \mathrm{~Gy}$. The dose rate dependence of the diamond detector response was measured in the range from 0.17 to $5.45 \mathrm{~Gy} / \mathrm{min}$, by irradiating the device in five steps, $1 \mathrm{~Gy}$ each, at different dose rates obtained varying the accelerator monitor unit rate. The adopted dose rate range corresponds to the whole monitor unit rate range available by the accelerator. The SCDD was placed in water at the depth of the dose maximum $\left(\mathrm{d}_{\max }=25 \mathrm{~mm}\right)$ and a Semiflex chamber was used to monitor the beam output. The diamond detector signal $\left(\mathrm{M}_{\mathrm{SCDD}}\right)$ was then divided by the corresponding signal from the Semiflex chamber $\left(\mathrm{M}_{\mathrm{SF}-\mathrm{IC}}\right)$. The percentage variation of the ratio $\mathrm{R}=\mathrm{M}_{\mathrm{SCDD}} / \mathrm{M}_{\mathrm{SF}-\mathrm{IC}}$ was then calculated with respect to the $\mathrm{R}$ value obtained: (i) at plateau in the preirradiation measurements, (ii) at delivered dose of $1 \mathrm{~Gy}$ in dose dependence measurements, and (iii) at the maximum dose rate in dose rate dependence investigation.

Depth dose distributions were measured along the central beam axis with the SCDD, the Advanced Markus ionization chamber, and the Si-D. Electron beams of 6, 8, 10, 12 and $15 \mathrm{MeV}$, with $6 \times 6,10 \times 10$, and $20 \times 20 \mathrm{~cm}^{2}$ square fields were investigated. All measured depth dose distributions were normalized to the dose at $\mathrm{d}_{\max }$ and the resulting percentage depth dose curves (PDD) from the SCDD detector were compared with those obtained by the ionization chamber and the silicon diode. No correction was applied to the SCDD and Si-D measured PDD curves, whereas depth ionization curves from the AM-IC were first corrected for ion recombination and polarity effects and then converted to depth dose curves. ${ }^{4}$ Ion recombination and polarity corrections at all measurement depths were obtained by interpolating correction factors measured near the phantom surface (depth of $5 \mathrm{~mm}$ ), at $\mathrm{d}_{\max }$, and at the depths of $80 \%$ and $50 \%$ of the dose maximum. The polarity correction was determined measuring the collected charge with positive and negative polarizing potential. The ion recombination correction was determined using the two voltage method ${ }^{4}$ with a voltage ratio of 3 .

In-plane and cross-plane lateral beam profiles were recorded with diamond detector, silicon diode, and Semiflex ionization chamber in $6 \times 6,10 \times 10$, and $20 \times 20 \mathrm{~cm}^{2}$ electron fields, at the respective depths of dose maximum, for all the available 6-15 MeV electron energies. The measured profiles were centered on the central axis in order to remove uncertainties in detector positioning in the scan direction and normalized to the dose at central axis. No further data manipulation was carried out. The profiles measured by the SCDD detector were compared with the ones obtained by the ionization chamber and the silicon diode in terms of $80 \%-20 \%$ penumbra and difference plots between profiles.

Parameters from both lateral beam profiles and percentage depth dose curves were determined by means of MEPHYSTO $\mathrm{mc}^{2}$ software (PTW-Freiburg) applying the IAEA TRS-398 procedures. Penumbra values were calculated for all the measured lateral beam profiles and reported as the arithmetic mean of the left and right penumbras. The main features derived by the PDD curves were the depths $R_{100}, R_{90}$, and $R_{50}$, the practical range $R_{p}$, the surface dose $D_{s}$, and the $x$-ray background.

Output factors (OF) were determined by means of the diamond detector, the Advanced Markus chamber, and the silicon diode with the $10 \mathrm{MeV}$ electron beam, for the field sizes $6 \times 6,10 \times 10,14 \times 14$, and $20 \times 20 \mathrm{~cm}^{2}$. The detectors were placed at a depth corresponding to the depth of dose maximum as determined by means of the Advanced Markus chamber for each field size. For a given beam energy, OFs are given by the ratio of the dose at $d_{\max }$ in the investigated nonreference field size to the dose at $d_{\max }$ in the reference field size (i.e., $10 \times 10 \mathrm{~cm}^{2}$ in this work) for the same number of monitor units. ${ }^{2-4}$ For the diamond detector and the silicon diode the OFs were determined as ratios of detector readings at $d_{\max }$ under nonreference and reference conditions. ${ }^{4}$ For the AM-IC the variation in water to air stopping-power ratio with depth was taken into account ${ }^{3,4}$ and the OFs were determined as

$$
\mathrm{OF}_{f}=\frac{M\left(d_{\max , f}\right)}{M\left(d_{\max , f_{o}}\right)} \frac{s_{\mathrm{w}, \operatorname{air}}\left(d_{\max , f}\right)}{s_{\mathrm{w}, \operatorname{air}}\left(d_{\max , f_{o}}\right)},
$$

where $M$ is the chamber reading corrected for the influence quantities, $s_{\mathrm{w}, \text { air }}$ is the water to air stopping power ratio, $d_{\text {max,f }}$ is the depth of dose maximum for the field size $f$, and $d_{\text {max }}$,fo is the depth of dose maximum for the reference field size $f_{o}$.

\section{RESULTS AND DISCUSSION}

\section{III.A. Preirradiation, dose, and dose rate dependence}

The preirradiation of the SCDD is shown in Fig. 3. Maximum variations within $0.7 \%$ can be observed with respect to the plateau value, while a signal stability within $0.1 \%(1 \sigma)$ is obtained after about 5 Gy. Such a signal stability is maintained during daily use with no need of further preirradiation procedures. 


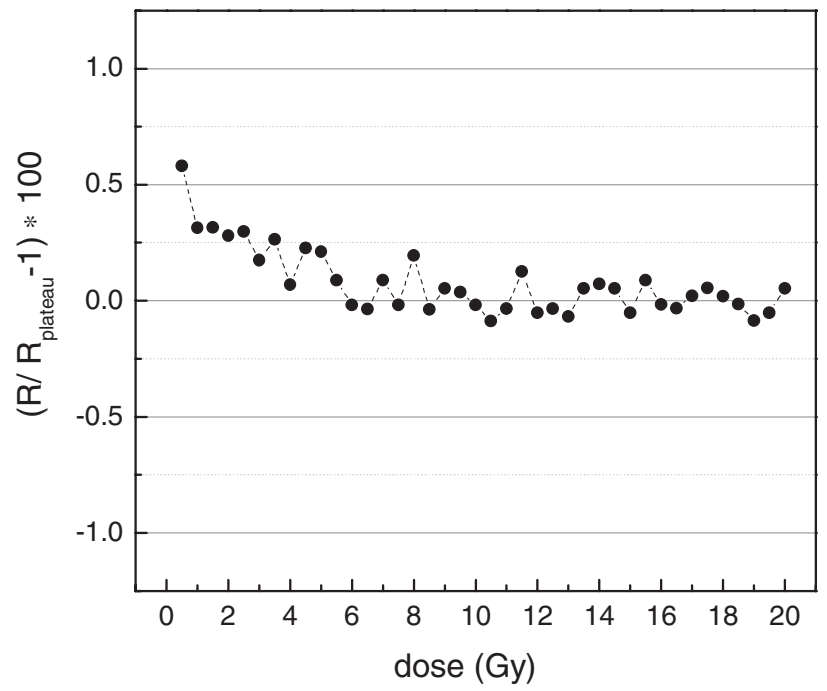

FIG. 3. Preirradiation of the diamond detector in a $10 \mathrm{MeV}, 10 \times 10 \mathrm{~cm}^{2}$ electron beam. The percentage deviation of the SCDD to SF-IC ratio $\mathrm{R}=\mathrm{M}_{\mathrm{SCDD}} / \mathrm{M}_{\mathrm{SF}-\mathrm{IC}}$ is reported as a function of the delivered dose and normalized to the plateau value (mean value of the detector response from 10 to 20 Gy).

The charge measured from the diamond detector as a function of the delivered dose is shown in Fig. 4(a). The $\mathrm{R}^{2}$ parameter of the linear best fit was found to be 1 with a precision of $10^{-7}$. A sensitivity of $1.134 \mathrm{nC} / \mathrm{Gy}$ for the SCDD was derived from the measured data as the slope of the linear fit. In order to highlight deviations from linearity, the normalized percentage

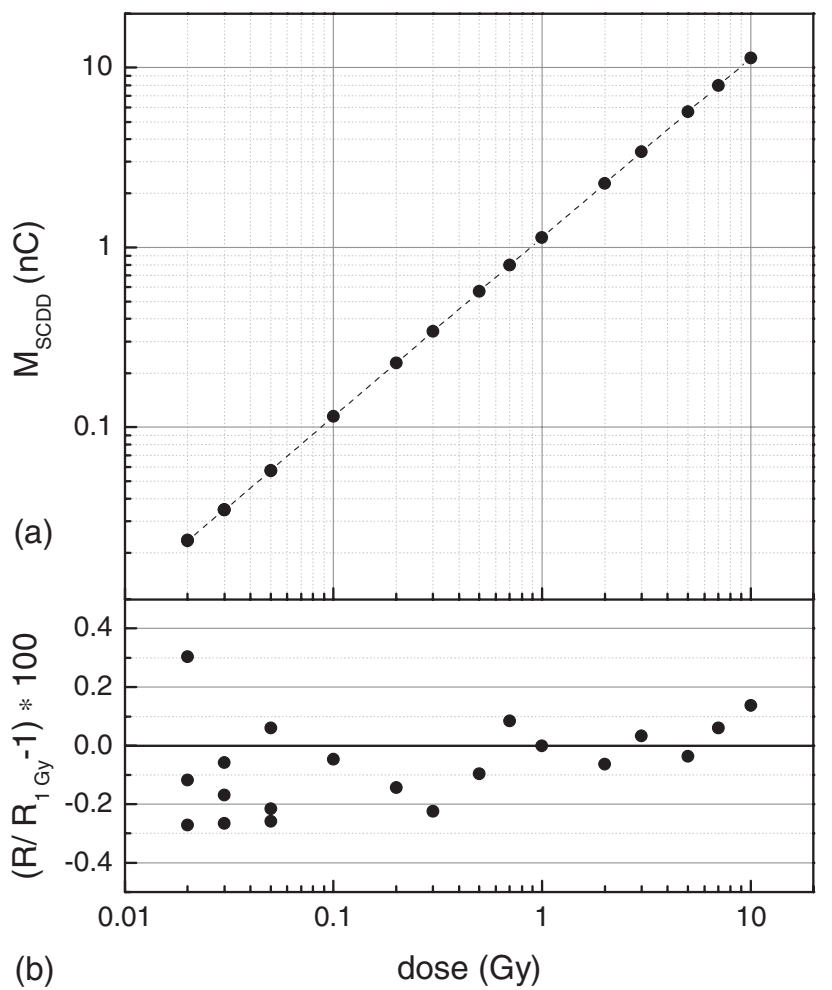

FIG. 4. (a) Measured SCDD charge as a function of delivered dose under irradiation with a $10 \mathrm{MeV}$ electron beam. (b) Percentage deviation of the ratio, $\mathrm{R}$, between SCDD and ionization chamber signal normalized to the value obtained with a delivered dose of $1 \mathrm{~Gy}$.

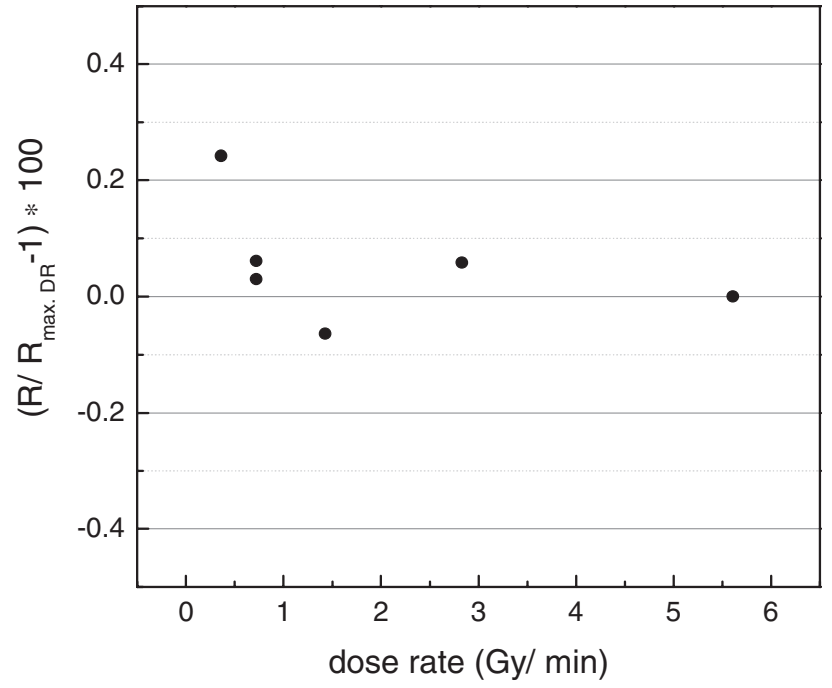

FIG. 5. Dose rate dependence of the SCDD response. The percentage deviation of the ratio $\mathrm{R}$ of SCDD to ionization chamber signal is reported, as a function of the dose rate. $\mathrm{R}$ values are normalized to the value obtained at the maximum dose rate.

ratio, $\mathrm{R}$, of the SCDD signal to the SF-IC signal is shown in Fig. 4(b) as a function of delivered dose. Deviations no larger than $\pm 0.3 \%$ can be observed down to the minimum delivered dose of 0.02 Gy. These deviations are within the experimental uncertainties taking into account that the typical relative standard deviation of the measured $\mathrm{R}$ values was $0.2 \%$.

The SCDD response as a function of dose rate is shown in Fig. 5 as percentage variation of the ratio between the SCDD and SF-IC signal. A maximum variation of $0.3 \%$ was found in the whole available dose rate range. Such a variation is consistent with the experimental uncertainties. No correction for dose rate dependence was applied to the measurements from the diamond detector in the dosimetric characterization discussed in Secs. III.B-III.D. However, it is worth to point out that the investigated dose rate range was spanned by varying the accelerator pulse repetition frequency, thus keeping constant the dose per pulse. As a consequence, the present results do not exclude a possible dose per pulse dependence of the SCDD response.

\section{III.B. Depth dose curves}

Central axis PDD curves measured by the SCDD, the AMIC, and the Si-D are reported in Fig. 6(a) for 6, 10, and $15 \mathrm{MeV}$ electron beams with field size $10 \times 10 \mathrm{~cm}^{2}$. In addition, difference plots between PDDs measured by SCDD and Si-D [Fig. 6(b)] as well as by SCDD and AM-IC [Fig. 6(c)] are reported. For all the energies, the PDD curves measured by the SCDD agree with those measured by the Si-D typically within $\pm 1 \%$ [see Fig. 6(b)]. A slightly worse agreement was found between SCDD and AM-IC, as shown in Fig. 6(c), with the largest differences corresponding to the steepest dose gradient region. In general, the percentage dose values measured by the plane-parallel ionization chamber tend to be higher in the build-up region and lower at depths beyond $d_{\max }$ when compared to the values measured 


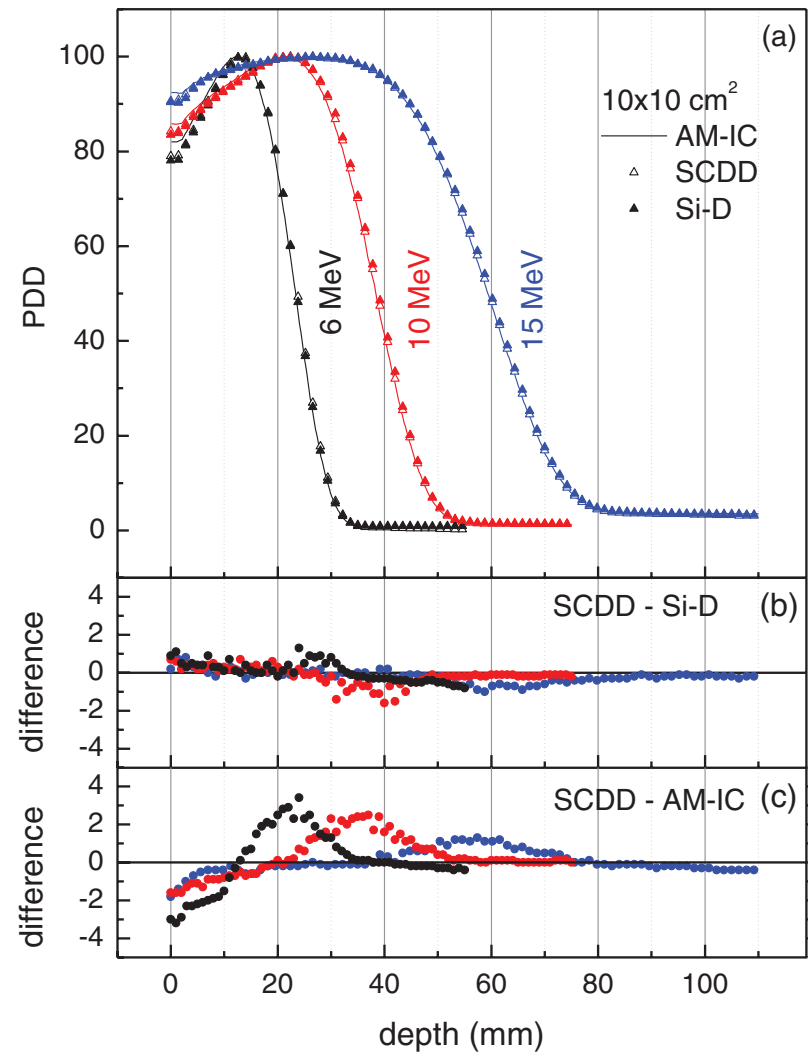

FIG. 6. Percentage depth dose curves for the SCDD, the Si-D, and the Advanced Markus chamber for 6,10 , and $15 \mathrm{MeV}, 10 \times 10 \mathrm{~cm}^{2}$ electron beams (a). Difference plots between PDDs from SCDD and Si-D (b) and SCDD and AM-IC (c) are also shown.

by both SCDD and Si-D. Recent papers ${ }^{33-36}$ showed that perturbation factors in electron beams can be nonunity also for "well-guarded" plane-parallel ionization chambers and their values depend on the measurement depth. Such a dependence should be accounted for when measuring depth dose distributions. Published Monte Carlo calculations and experimental data indicate that an appropriate shift of the chamber effective point of measurement allows to minimize the effect of perturbation factors on the measured PDD curves. ${ }^{35,37}$ Although the most published data refer to the NACP and Roos plane parallel chambers, Wang and Rogers ${ }^{36}$ in their Monte Carlo study on replacement correction factor, $\mathrm{p}_{\text {repl }}$, included calculations for the Advanced Markus chamber in $6 \mathrm{MeV}$ electron beam. Their results show a depth dependence of $\mathrm{p}_{\text {repl }}$ factor for the
AM-IC similar to that obtained for the Roos chamber. Thus, the systematic differences observed between the AM-IC PDD curves and those obtained by the SCDD and Si-D could be ascribed to a variation with measurement depth of the AM-IC overall perturbation factor. Accordingly, a shift of the AM-IC effective point of measurement $0.4 \mathrm{~mm}$ toward the air cavity drastically reduces the differences among PDD curves by the three detectors for all energies and field sizes. Nevertheless, it is worth to point out that the observed differences in the PDD curves do not significantly affect the values of dosimetric parameters like the beam quality specifier, $\mathrm{R}_{50}$, and the depth of dose maximum, $\mathrm{R}_{100}$, as can be seen in Table I, where the main features of the PDD curves shown in Fig. 6(a) are reported. As an example, the largest difference among the measured $R_{50}$ values in Table $I$ is $0.5 \mathrm{~mm}$ and such an uncertainty in the value of the beam quality specifier corresponds to a relative uncertainty component of less than $0.1 \%$ in the reference absorbed dose to water determination.

\section{III.C. Profiles}

The normalized cross-plane profiles measured by the SCDD, the Si-D, and the SF-IC in $6 \times 6,10 \times 10$, and $20 \times 20 \mathrm{~cm}^{2}$ field size and under $6 \mathrm{MeV}$ electron beam irradiation are shown in Fig. 7. Difference plots between profiles measured by SCDD and Si-D (indicated as SCDD - Si-D) and by SCDD and SF-IC (SCDD - SF-IC) are also shown for the investigated field sizes. Both the SCDD and the Si-D were placed in vertical orientation, i.e., with the circular 3.8 and 1 $\mathrm{mm}^{2}$ electrodes defining their active surface area facing the impinging electron beam. As recommended by the manufacturer, the SF-IC was positioned horizontally, i.e., with its stem axis perpendicular to the scan direction; thus, the diameter of the active area along the scan direction is $5.5 \mathrm{~mm}$. Differences between the spatial resolution of the investigated SCDD and that of the reference dosimeters can be easily noticed from the difference plots. The observed symmetric peaks, with respect to the central beam axis, clearly indicate a progressive penumbra sharpening, i.e., an improvement in spatial resolution, passing from the SF-IC to the SCDD and from the SCDD to the Si-D. The silicon diode provides a slightly steeper profile curve in the penumbra region with respect to the diamond detector, thus giving rise to small deviations in the SCDD Si-D difference plots. Similar trend but with opposite sign is observed in the SCDD - SF-IC plots, thus indicating sharper

TABLE I. PDD analysis parameters for the depth dose curves reported in Fig. 6.

\begin{tabular}{|c|c|c|c|c|c|c|c|c|c|}
\hline \multirow{2}{*}{$\begin{array}{l}10 \times 10 \mathrm{~cm}^{2} \\
\text { PDD analysis }\end{array}$} & \multicolumn{3}{|c|}{$6 \mathrm{MeV}$} & \multicolumn{3}{|c|}{$10 \mathrm{MeV}$} & \multicolumn{3}{|c|}{$15 \mathrm{MeV}$} \\
\hline & SCDD & Si-D & AM-IC & SCDD & Si-D & AM-IC & SCDD & Si-D & AM-IC \\
\hline $\mathrm{R}_{100}(\mathrm{~mm})$ & 13.0 & 13.0 & 12.2 & 21.1 & 22.0 & 21.1 & 26.0 & 26.0 & 26.1 \\
\hline $\mathrm{R}_{90}(\mathrm{~mm})$ & 17.8 & 17.8 & 17.3 & 29.9 & 30.1 & 29.3 & 44.7 & 44.8 & 44.4 \\
\hline $\mathrm{R}_{50}(\mathrm{~mm})$ & 23.7 & 23.6 & 23.3 & 38.7 & 38.9 & 38.4 & 59.7 & 59.9 & 59.4 \\
\hline $\mathrm{R}_{\mathrm{p}}(\mathrm{mm})$ & 29.8 & 29.6 & 29.4 & 47.5 & 47.8 & 47.4 & 72.9 & 72.9 & 72.6 \\
\hline $\mathrm{D}_{\mathrm{s}}(\%)$ & 78.9 & 77.9 & 82.0 & 84.2 & 83.5 & 85.8 & 90.7 & 90.4 & 92.4 \\
\hline X-ray Bck. (\%) & 0.4 & 1.5 & 0.6 & 1.4 & 1.5 & 1.3 & 4.3 & 3.9 & 3.7 \\
\hline
\end{tabular}




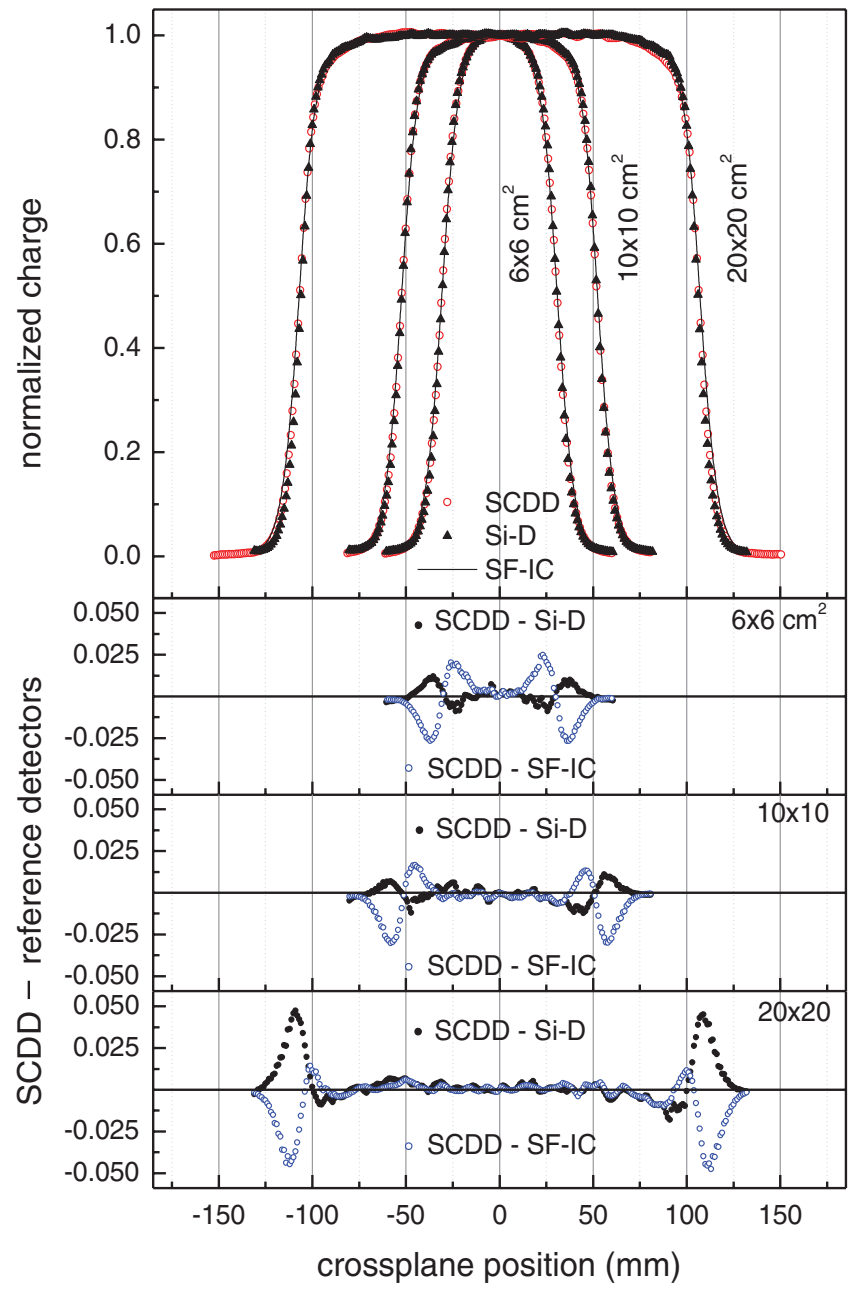

FIG. 7. Normalized cross-plane profiles of $6 \mathrm{MeV}$ and $6 \times 6,10 \times 10$, and $20 \times 20 \mathrm{~cm}^{2}$ electron beams, measured at depth of dose maximum by the SCDD, the Si-D, and the SF-IC. The difference plots between profiles by diamond and both reference detectors are also shown.

profile measured by the SCDD. Results analogous to those shown in Fig. 7 were obtained for the in-plane profiles and for all the electron beam energies investigated.

The $80 \%-20 \%$ penumbra widths as derived by the SCDD, the Si-D, and the SF-IC cross-plane profiles are re- ported in Table II for the investigated field sizes and the 6, 10,12 , and $15 \mathrm{MeV}$ nominal electron energies. Similar results were obtained from in-plane profiles. The silicon diode exhibits the narrowest measured penumbras with an average difference of $0.4 \mathrm{~mm}$ with respect to those measured by the diamond detector. The ionization chamber shows the lowest spatial resolution, with an average penumbra broadening of 1.8 and $1.4 \mathrm{~mm}$ with respect to the Si-D and the SCDD values, respectively. Such differences in spatial resolution among the detectors, either under photon and electron beam irradiation, can be ascribed to variations in the geometry and size of the

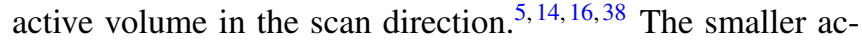
tive sizes of the diamond detector and of the silicon diode lead to a better spatial resolution and to smaller measured penumbras than those from the SF-IC, which is well known to be unsuitable for precise penumbra determination.

According to literature, ${ }^{5,16,26,38}$ the spatial resolution of the SCDD is improved by measuring lateral beam profiles with the detector placed in horizontal orientation and with its long axis in the scan direction (see Fig. 2, horizontal crossplane). In this case, the thin active volume of the SCDD lies in a plane perpendicular to the scan direction and the effective detector size along this scan direction corresponds to the thickness $(\sim 1 \mu \mathrm{m})$ of the active volume. An improvement in spatial resolution is thus expected in such conditions, with respect to the vertical orientation. In-plane profiles were also recorded, with the SCDD in the very same horizontal orientation (see Fig. 2, horizontal in-plane). In this configuration, the active volume is coplanar with the scanning direction and the effective detector size corresponds to the diameter of the circular $\mathrm{Al}$ electrode $(2.2 \mathrm{~mm})$. Therefore, penumbra values similar to those obtained with the SCDD in vertical orientation are expected.

The values of $80 \%-20 \%$ penumbras for the all measured profiles are reported in Table III. As predicted by the above mentioned geometrical considerations, the SCDD showed the highest spatial resolution in cross-plane profiles and horizontal orientation. In this configuration the SCDD and the SiD profiles are nearly identical, i.e., no peaks structures are visible in the difference plots between their respective lateral beam profiles, and the penumbra values agree within $0.2 \mathrm{~mm}$ (see Table III).

TABLE II. $80 \%-20 \%$ penumbra values derived from the cross-plane profiles by SCDD, Si-D, and SF-IC for various electron energies and field sizes.

\begin{tabular}{|c|c|c|c|c|c|c|c|}
\hline \multirow{2}{*}{$\begin{array}{l}6 \mathrm{MeV} \\
\text { Field size }\left(\mathrm{cm}^{2}\right)\end{array}$} & \multicolumn{3}{|c|}{$80 \%-20 \%$ Penumbra $(\mathrm{mm})$} & \multirow{2}{*}{$\frac{10 \mathrm{MeV}}{\text { Field size }\left(\mathrm{cm}^{2}\right)}$} & \multicolumn{3}{|c|}{$80 \%-20 \%$ Penumbra $(\mathrm{mm})$} \\
\hline & SCDD & Si-D & SF-IC & & SCDD & Si-D & SF-IC \\
\hline $6 \times 6$ & 11.3 & 10.9 & 12.6 & $6 \times 6$ & 11.6 & 11.1 & 12.9 \\
\hline $10 \times 10$ & 11.3 & 10.9 & 12.6 & $10 \times 10$ & 11.8 & 12.0 & 13.8 \\
\hline $20 \times 20$ & 11.5 & 10.6 & 13.1 & $20 \times 20$ & 12.4 & 12.1 & 14.3 \\
\hline $12 \mathrm{MeV}$ & \multicolumn{3}{|c|}{$80 \%-20 \%$ Penumbra $(\mathrm{mm})$} & $15 \mathrm{MeV}$ & \multicolumn{3}{|c|}{$80 \%-20 \%$ Penumbra $(\mathrm{mm})$} \\
\hline Field size $\left(\mathrm{cm}^{2}\right)$ & SCDD & Si-D & SF-IC & Field size $\left(\mathrm{cm}^{2}\right)$ & SCDD & Si-D & SF-IC \\
\hline $6 \times 6$ & 11.6 & 11.1 & 12.9 & $6 \times 6$ & 9.3 & 8.4 & 9.7 \\
\hline $10 \times 10$ & 11.3 & 11.5 & 13.2 & $10 \times 10$ & 9.5 & 9.4 & 10.8 \\
\hline $20 \times 20$ & 12.7 & 12.2 & 14.3 & $20 \times 20$ & 10.7 & 10.5 & 12.3 \\
\hline
\end{tabular}


TABLE III. $80 \%$ - $20 \%$ penumbra values derived from the lateral beam profiles obtained by the SCDD with scanning procedures and detector orientations illustrated in Fig. 2. For comparison, the penumbra values obtained by the $\mathrm{Si}-\mathrm{D}$ are also shown.

\begin{tabular}{lccc}
\hline \hline $6 \mathrm{MeV} 10 \times 10 \mathrm{~cm}^{2}$ & \multicolumn{3}{c}{$80 \%-20 \%$ Penumbra $(\mathrm{mm})$} \\
\cline { 2 - 4 } Scan direction & SCDD vertical & SCDD horizontal & Si-D \\
\hline Cross-plane & 11.3 & 10.7 & 10.9 \\
In-plane & 11.3 & 11.0 & 10.9 \\
\hline \hline
\end{tabular}

It should be pointed out that a small spatial resolution improvement, with respect to the SCDD in vertical orientation, is also observed for the in-plane profiles. Also in this configuration, the SCDD spatial resolution is very similar to that of the Si-D within $0.1 \mathrm{~mm}$ (see Table III). Such a behavior was also observed in a previous study carried out with a similar SCDD on clinical radiation therapy photon beams. ${ }^{26}$ Further work and Monte Carlo simulation have been planned to better clarify this aspect.

\section{III.D. Output factors}

Output factors are reported in Fig. 8 for the SCDD, the Si-D, and the AM-IC. A particular energy-dependent jaw setting is automatically set by the accelerator for each electron applicator in order to minimize the collimation scattering. Such changes in collimator settings determine a nonmonotonic variation of OFs with the applicator size, ${ }^{2}$ as clearly visible in Fig. 8 for all the detectors. It is worth to point out that the OFs calculated for SCDD and the reference detectors are compatible within the measurement uncertainties, being the typical measurement reproducibility $0.2 \%(1 \sigma)$.

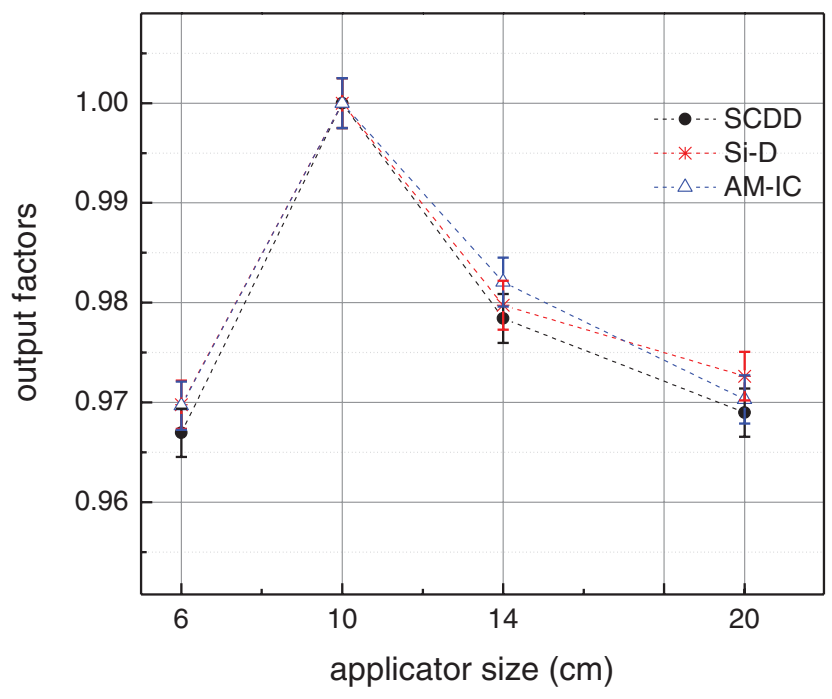

FIG. 8. Output factors vs applicator size measured by SCDD, Si-D, and AMIC in a $10 \mathrm{MeV}$ electron beam. Uncertainty bars are the experimental standard uncertainties.

\section{SUMMARY AND CONCLUSIONS}

The suitability of a small volume synthetic single crystal diamond detector for dosimetry in clinical electron beams was investigated. The proposed device, a synthetic diamond based Schottky diode, was encapsulated in the very same housing of a PTW-Freiburg silicon diode E detector and operated at zero bias voltage. Measurements were performed under 6, 8, 10, 12 , and $15 \mathrm{MeV}$ electron beams by a linear accelerator and field sizes from $6 \times 6$ to $20 \times 20 \mathrm{~cm}^{2}$ were used, shaped by standard applicators. Measurement results from the diamond detector were compared to those from PTW reference detectors routinely used for therapeutic electron beam dosimetry (Advanced Markus plane-parallel ionization chamber, Semiflex cylindrical ionization chamber, and Dosimetry Diode E $p$-type silicon detector). A maximum signal variation of about $0.7 \%$ was observed during the preirradiation, with a final stability of $0.1 \%(1 \sigma)$ after about $5 \mathrm{~Gy}$. Dose dependence tests evidenced a very good linearity with dose, with a deviation from linearity less than $\pm 0.3 \%$ down to the lowest delivered dose value of $0.02 \mathrm{~Gy}$. A negligible dose rate dependence was observed as well, with variations in the response of less than $0.3 \%$ in the dose rate range from 0.17 to $5.45 \mathrm{~Gy} / \mathrm{min}$. Depth dose curves showed an overall good agreement among detectors. Diamond detector and silicon diode provided a direct measurement of relative dose, whereas depth-dependent corrections were needed for the Advanced Markus plane parallel ionization chamber. Spatial resolution was evaluated by means of lateral beam profile measurements. The obtained results were found to well agree with the geometrical features of the tested detectors. Due to its small sensitive volume, the SCDD exhibits a better spatial resolution than the cylindrical ionization chamber. On the other hand, the silicon diode showed a slightly better resolution than diamond detector, when both of them are irradiated in vertical orientation (i.e., detector longitudinal axis parallel to the beam axis). However, a good improvement in the diamond detector spatial resolution was observed by performing profile measurements with the device oriented with its longitudinal axis perpendicular to the beam direction and parallel to the scan direction. In this case no differences can be noticed between the spatial resolution of the diamond and the silicon diode detectors. Output factors measured by diamond detector match the ones by a silicon diode and an Advanced Markus plane-parallel chamber within the measurement uncertainties.

From the results discussed above, and in particular due to its high spatial resolution, comparable to that of commercial silicon diode, and to a superior tissue equivalence, the tested diamond detector appears to be a good candidate for relative dosimetry in megavoltage clinical electron beams. Work is in progress to assess the suitability of the proposed diamond detector also for relative dosimetry in small or irregularly shaped electron beams.

\section{ACKNOWLEDGMENTS}

The authors wish to thank "Fondazione Roma" for financial support and PTW-Freiburg for providing the 
encapsulation kit used for the dosimeter investigated in the present paper and for helpful discussion. This work was also jointly supported by the EMRP participating countries within Euramet and the European Union.

a) Author to whom correspondence should be addressed. Electronic mail: gianluca.verona.rinati@uniroma2.it

${ }^{1}$ ICRU (International Commission on Radiation Units and Measurements), "Radiation dosimetry: Electron beams with energies between 1 and 50 MeV,'” ICRU Report No. 35 (ICRU, Bethesda, MD, 1984).

${ }^{2}$ F. M. Khan, K. P. Doppke, K. R. Hogstrom, G. J. Kutcher, R. Nath, S. C. Prasad, J. A. Purdy, M. Rozenfeld, and B. L. Werner, "Clinical electron beam dosimetry: Report of AAPM Radiation Therapy Committee Task Group No. 25,” Med. Phys. 18, 73-109 (1991)

${ }^{3}$ D. I. Thwaites, A. R. DuSautoy, T. Jordan, M. R. McEwen, A. Nisbet, A. E. Nahum, and W. G. Pitchford, "The IPEM code of practice for electron dosimetry for radiotherapy beams of initial energy from 4 to $25 \mathrm{MeV}$ based on an absorbed dose to water calibration," Phys. Med. Biol. 48, 2929-2970 (2003).

${ }^{4}$ IAEA (International Atomic Energy Agency), "Absorbed dose determination in external beam radiotherapy: An international code of practice for dosimetry based on standards of absorbed dose to water," Technical Report Series No. 398 (IAEA, Vienna, 2000).

${ }^{5}$ M. Heydarian, P. W. Hoban, W. A. Beckham, I. M. Borchardt, and A. H. Beddoe, "Evaluation of a PTW diamond detector for electron beam measurements," Phys. Med. Biol. 38, 1035-1042 (1993).

${ }^{6} \mathrm{G}$. Rikner, "Characteristics of a p-Si detector in high energy electron fields," Acta Radiol. Oncol. 24, 71-74 (1985).

${ }^{7}$ J. N. Eveling, A. M. Morgan, and W. G. Pitchford, "Commissioning a ptype silicon diode for use in clinical electron beams," Med. Phys. 26, 100107 (1999).

${ }^{8}$ I. Griessbach, M. Lapp, J. Bohsung, G. Gademann, and D. Harder, "Dosimetric characteristics of a new unshielded silicon diode and its application in clinical photon and electron beams," Med. Phys. 32, 3750-3754 (2005).

${ }^{9}$ ICRU (International Commission on Radiation Units and Measurements), "Stopping powers for electrons and positrons," ICRU Report No. 37 (ICRU, Bethesda, MD, 1984).

${ }^{10}$ H. Song, M. Ahmad, J. Deng, Z. Chen, N. J. Yue, and R. Nath, "Limitations of silicon diodes for clinical electron dosimetry,” Radiat. Prot. Dosim. 120, 56-59 (2006).

${ }^{11}$ S. F. Kozlov, "Preparation and characteristics of natural diamond nuclear radiation detectors," IEEE Trans. Nucl. Sci. 22, 160-170 (1975).

${ }^{12}$ R. J. Keddy, T. L. Nam, and R. C. Burns, "The detection of ionizing radiation by natural and synthetic diamond crystals and their application as dosimeters in biological environments," Carbon 26, 345-356 (1988).

${ }^{13}$ B. Planskoy, "Evaluation of diamond radiation dosimeters," Phys. Med. Biol. 25, 519-532 (1980).

${ }^{14} \mathrm{~S}$. Vatnitsky and H. Jarvinen, "Application of a natural diamond detector for the measurement of relative dose distributions in radiotherapy," Phys. Med. Biol. 38, 173-184 (1993).

${ }^{15}$ D. G. van der Merwe and R. J. Keddy, "Relative electron dosimetry using a synthetic diamond probe," Rad. Phys. Chem. 54, 325-334 (1999).

${ }^{16} \mathrm{~S}$. N. Rustgi, "Evaluation of the dosimetric characteristics of a diamond detector in photon beam measurements," Med. Phys. 22, 567-570 (1995).

${ }^{17}$ W. U. Laub, T. W. Kaulich, and F. Nüsslin, "A diamond detector in the dosimetry of high-energy electron and photon beams," Phys. Med. Biol. 44, 2183-2192 (1999).

${ }^{18}$ P. N. Mobit and G. A. Sandison, "An EGS4 Monte Carlo examination of the response of a PTW-diamond radiation detector in megavoltage electron beams," Med. Phys. 26, 839-844 (1999).

${ }^{19}$ P. Björk, T. Knöös, and P. Nilsson, "Comparative dosimetry of diode and diamond detectors in electron beams for intraoperative radiation therapy," Med. Phys. 27, 2580-2588 (2000).

${ }^{20}$ C. De Angelis, S. Onori, M. Pacilio, G. A. Cirrone, G. Cuttone, L. Raffaele, M. Bucciolini, and S. Mazzocchi, "An investigation of the operating char- acteristics of two PTW diamond detectors in photon and electron beams," Med. Phys. 29, 248-254 (2002).

${ }^{21}$ M. Bucciolini, F. Banci Buonamici, S. Mazzocchi, C. De Angelis, S. Onori, and G. A. P. Cirrone, "Diamond detector versus silicon diode and ion chamber in photon beams of different energy and field size," Med. Phys. 30, 2149-2154 (2003).

${ }^{22}$ N. Ade, T. L. Nam, and M. Assiamah, "A synthetic diamond probe for both low-energy mammography $\mathrm{x}$-rays and high-energy electron therapy beams," Radiat. Phys. Chem. 81, 232-239 (2012).

${ }^{23}$ A. Fidanzio, L. Azario, R. Kalish, Y. Avigal, G. Conte, P. Ascarelli, and A. Piermattei, "A preliminary dosimetric characterization of chemical vapor deposition diamond detector prototypes in photon and electron radiotherapy beams," Med. Phys. 32, 389-395 (2005).

${ }^{24}$ S. Almaviva, M. Marinelli, E. Milani, A. Tucciarone, G. Verona-Rinati, R. Consorti, A. Petrucci, F. De Notaristefani, and I. Ciancaglioni, "Synthetic single crystal diamond diodes for radiotherapy dosimetry," Nucl. Instrum. Methods Phys. Res. A 594, 273-277 (2008).

${ }^{25}$ S. Almaviva, I. Ciancaglioni, R. Consorti, F. De Notaristefani, C. Manfredotti, M. Marinelli, E. Milani, A. Petrucci, G. Prestopino, C. Verona, and G. Verona-Rinati, "Synthetic single crystal diamond dosimeters for intensity modulated radiation therapy applications," Nucl. Instrum. Methods Phys. Res. A 608, 191-194 (2009).

${ }^{26}$ I. Ciancaglioni, Marco Marinelli, E. Milani, G. Prestopino, C. Verona, G. Verona-Rinati, R. Consorti, A. Petrucci, and F. De Notaristefani, "Dosimetric characterization of a synthetic single crystal diamond detector in clinical radiation therapy small photon beams," Med. Phys. 39, 4493-4502 (2012).

${ }^{27}$ M. Pimpinella, I. Ciancaglioni, R. Consorti, C. Di Venanzio, A. S. Guerra, A. Petrucci, A. Stravato, and G. Verona-Rinati, "A synthetic diamond detector as transfer dosimeter for $D_{w}$ measurements in photon beams with small field sizes," Metrologia 49, S207-S210 (2012).

${ }^{28}$ J. Pearce, R. Thomas, and A. DuSautoy, "The characterization of the Advanced Markus ionization chamber for use in reference electron dosimetry in the UK," Phys. Med. Biol. 51, 473-483 (2006).

${ }^{29}$ S. Almaviva, M. Marinelli, E. Milani, G. Prestopino, A. Tucciarone, C. Verona, G. Verona-Rinati, M. Angelone, M. Pillon, I. Dolbnya, K. Sawhney, and N. Tartoni, "Chemical vapor deposition diamond based multilayered radiation detector: Physical analysis of detection properties," J. Appl. Phys. 107, 014511-014517 (2010).

${ }^{30}$ PTW Freiburg (private communication).

${ }^{31}$ M. R. McEwen, A. J. Williams, and A. R. DuSautoy, "Determination of absorbed dose calibration factors for therapy level electron beam ionization chambers," Phys. Med. Biol. 46, 741-755 (2001).

${ }^{32}$ I. J. Das, C. W. Cheng, R. J. Watts, A. Ahnesjö, J. Gibbons, X. A. Li, J. Lowenstein, R. K. Mitra, W. E. Simon, and T. C. Zhu, "Accelerator beam data commissioning equipment and procedures: Report of the TG-106 of the Therapy Physics Committee of the AAPM," Med. Phys. 35, 4186-4215 (2008).

${ }^{33}$ E. Chin, H. Palmans, D. Shipley, M. Bailey, and F. Verhaegen, "Analysis of dose perturbation factors of a NACP-02 ionization chamber in clinical electron beams," Phys. Med. Biol. 54, 307-326 (2009).

${ }^{34} \mathrm{~F}$. Araki, "Monte Carlo calculations of correction factors for plane-parallel ionization chamber in clinical electron dosimetry," Med. Phys. 35, 40334040 (2008)

${ }^{35} \mathrm{~K}$. Zink and J. Wulff, "Positioning of a plane-parallel ionization chamber in clinical electron beams and the impact on perturbation factors," Phys. Med. Biol. 54, 2421-2435 (2009).

${ }^{36}$ L. L. W. Wang and W. O. Rogers, "Replacement correction factors for plane-parallel ion chambers in electron beams," Med. Phys. 37, 461-465 (2010).

${ }^{37}$ H. K. Looe, D. Harder, and B. Poppe, "Experimental determination of the effective point of measurement for various detectors used in photon and electron beam dosimetry," Phys. Med. Biol. 56, 4267-4290 (2011).

${ }^{38}$ W. U. Laub and T. Wong, "The volume effect of detectors in the dosimetry of small fields used in IMRT," Med. Phys. 30, 341-347 (2003). 